\title{
Phenotype Changes of Circulating Monocytes in a Hypercholesterolemic Swine Model of Coronary Artery Disease
}

\author{
Silverio Sbrana ${ }^{1^{*}}$, Gualtiero Pelosi ${ }^{2}$, Maria Rita Puntoni ${ }^{3}$, Federica Viglione ${ }^{2}$, Maria Giovanna Trivella ${ }^{2}$ and Oberdan Parodi ${ }^{2}$ \\ ${ }^{1}$ Flow Cytometry Laboratory, CNR Institute of Clinical Physiology, Massa, Italy \\ ${ }^{2}$ Laboratory of Experimental Cardiology, CNR Institute of Clinical Physiology, Pisa, Italy \\ ${ }^{3}$ Laboratory of Dyslipidemic Diseases and Atherosclerosis, CNR Institute of Clinical Physiology, Pisa, Italy
}

"Correspondig author: Silverio Sbrana, Flow Cytometry Laboratory, CNR Institute of Clinical Physiology, "G. Pasquinucci” Heart Hospital, 54100 Massa (MS), Italy, Tel: +39-0585-493595; Fax number: +39-0585-493601; E-mail: silverio.sbrana@ifc.cnr.it

Rec date: Jun 16, 2014, Acc date: Aug 21, 2014, Pub date: Aug 23, 2014

Copyright: (C) 2014 Sbrana S, et al. This is an open-access article distributed under the terms of the Creative Commons Attribution License, which permits unrestricted use, distribution, and reproduction in any medium, provided the original author and source are credited.

\begin{abstract}
Objective: Inflammation and immunity activation play a key role in atherosclerosis (ATS) onset and progression. Aim of this study was to investigate the relationships between phenotype of circulating monocytes and coronary artery disease (CAD) development in a histologically well-characterized swine model of ATS.

Methods:Blood samples were obtained from 6 animals at baseline and after 16 weeks high fat cholesterolenriched diet. Flow cytometry monocyte identification was performed (CD172a marker). Adhesion (CD18a, CD11a, CD11R3, CD49d, CD29), differentiation (CD14) and activation receptors (SLA-DR, CD16, CD163) were quantified as percentage of positivity (\%) and Relative Fluorescence Intensity (RFI). Lipid parameters (LDL, oxLDL, HDL) and soluble endothelial ICAM-1 were measured and histologic quantitative assessment of coronary ATS was performed.
\end{abstract}

Results:Flow cytometry analysis demonstrated a significant post-diet decrease of CD14 RFI and an increment of $\%$ SLA-DR. Pre-diet values of ICAM-1 and \% SLA-DR correlated reciprocally ( $P=0.0191)$ and with several CAD severity indexes $(P \leq 0.02)$. Positive correlations between RFI changes of CD29 $(P=0.0213)$ and $C D 18 a(P=0.0341)$ and morphometric indexes of coronary ATS were found. Post-diet RFI values of CD29, CD18a and CD16 were also closely related to morphometric parameters $(\mathrm{P}<0.03)$. A cumulative post-diet tendency to increase of CD14 low/ CD163 ${ }^{\text {high }}$ monocyte fraction $(45.07 \pm 2.27$ vs. $40.14 \pm 3.16)$ and a tight correlation between changes of this monocyte subset and corresponding HDL variations $(P=0.0100)$ were also observed.

Conclusions:Blood monocyte orientation towards a macrophage-like phenotype, similar to a HDL-induced maturation, and a close association between markers changes and severity of diet induced coronary ATS could provide new insights into plaque growth and progression in CAD.

Keywords: Monocyte; Swine; Peripheral blood; Flow cytometry; Hypercholesterolemic diet; Coronary atherosclerosis; Histopathology

\begin{abstract}
Abbreviations:
ATS: Atherosclerosis; HCD: Hypercholesterolemic Diet; CAD: Coronary Artery Disease

\section{Introduction}

Atherosclerosis (ATS) is a chronic inflammatory process of large and medium-size arteries, in which the focal endothelial activation, induced by hypercholesterolemia and lipoproteins deposition in the arterial intima, represents an essential initiating event $[1,2]$. The endothelial activation determines an increased adhesion molecule expression and chemokine production, which mediate the initial rolling of blood leukocytes on the endothelial vascular surface, their subsequent firm adhesion at activation sites, and their final recruitment into the underlying intima [3,4]. Moreover, hemodynamic flow patterns, typical of ATS-prone segments (low or oscillatory shear stress), give rise to an increased expression of adhesion molecule and inflammatory genes by endothelial cells and, together with lipoprotein
\end{abstract}

accumulation, aggravate intimal inflammation [5]. Among blood leukocyte subsets, the study of monocyte migration and monocytederived macrophage accumulation into arterial wall, in both early atherogenesis and advanced plaque progression, has become a topic of great interest in the last decade [6-8]. In particular, immunohistochemical analysis of human atherosclerotic lesions, as well as experimental studies in gene-targeted mice, suggested that the monocyte integrins VLA-4 (very late antigen-4) and LFA-1 (lymphocyte function-associated antigen-1) and their corresponding endothelial cell ligands, VCAM-1 (vascular cell adhesion molecule-1) and ICAM-1 (intercellular adhesion molecule-1), are particularly important in plaque initiation [7]. Furthermore, the interest concerning the role of different monocyte subsets in ATS initiation and progression has arisen due to the demonstration of phenotypic and functional heterogeneity of circulating monocytes in mice, humans and other species [8-10]. Two major subsets of blood monocytes have been described based on differential expression of CCR1, CCR2 and CX3CR1 (fractalkine receptor) chemokine receptors [11]. The two murine monocyte subsets, classical (referred as Ly6 $\mathrm{C}^{\text {high }}$ ) and non-classical (referred as Ly6Clow) are homologous to the human classical (CD14 $\left.4^{\text {high }} / \mathrm{CD} 16^{\text {neg }}\right)$ and non-classical $\left(C D 14^{\text {low }}\right.$ CD16 $\left.{ }^{\text {high }}\right)$ monocyte subsets [10]. In hypercholesterolemic mice the 
monocytosis is dominated by the classical subset and correlates with atherosclerotic plaque size and foam cell content [12]. However, there are important differences in the characterization and role of monocyte subset and function in humans as compared to mice. Therefore, the choice of the animal model that more accurately reflects the process of atherogenesis in humans is mandatory. With the completion of the genome sequence and the characterization of many regulatory molecules and markers, the pig has emerged as a predictive experimental model for the study of human innate immunity in several inflammatory pathologies, overcoming the limitations of data derived from rodents [13]. A previous immunohistochemical study demonstrated the translational potential towards human pathology of a swine model of vascular damage associated with mild hypercholesterolemia [14]. Other histological studies in pigs showed that experimentally induced hypercholesterolemia produces coronary endothelial dysfunction with intimal hyperplasia by accumulation of lipids and foam cells in the vascular wall $[15,16]$. Moreover, atherogenic diet has been shown to increase the level of cholesterol efflux transporter ABCA1 in pig blood monocytes prior to atherosclerotic lesion formation, and higher ABCA1 levels are associated with higher plaque complexity [17].

The aim of this study has been to evaluate by flow cytometry the phenotype changes of circulating monocytes in a porcine model of coronary artery disease (CAD) induced by a hypercholesterolemic diet (HCD) and to investigate, for the first time, their relationships with the degree of coronary ATS development measured by comprehensive hystomorphometric analysis.

\section{Materials and Methods}

\section{Animals and Diet}

Six male domestic pigs (mean body weight $36 \pm 2 \mathrm{Kg}$ at baseline) were purchased from a commercial breeder. Animal instrumentation and experimental protocol were approved by the Animal Care Committee of the Italian Ministry of Health and were in accordance with the Italian Law (DL-116, Jan. 27, 1992), which is in compliance with the National Institute of Health publication Guide for the Care and Use of Laboratory Animals. Pigs were fed for 4 months with a high-lipid diet containing lard (20\%), cholesterol (4\%), oil (3\%), fibers (7\%), sodium cholate (1.5\%), besides proteins (17.5\%) and carbohydrates (33\%). The total energy from the HCD was 4450 $\mathrm{Kcal} / \mathrm{Kg}$ with a fatty energy fraction corresponding to $2430 \mathrm{Kcal} / \mathrm{Kg}$ (54.6\% of the total energy contribution). Both at baseline (pre-diet) and at the end of the study (post-diet), each animal underwent blood sampling for monocyte flow cytometry analysis. At the end of diet period, animals were sacrificed under anaesthesia (intramuscolar administration of $10 \mathrm{mg} / \mathrm{kg}$ of Zoletil ${ }^{\circledR}, 0.05 \mathrm{mg} / \mathrm{kg}$ atropine and maintained with isofluorane, together with $5 \mathrm{mg} / \mathrm{kg} / \mathrm{hour}$ of propofol intravenous infusion); the whole heart was harvested for histology and immunohistochemistry processing of coronary arteries.

\section{Histology and Morphometry}

Consecutive coronary segments, $0.5 \mathrm{~cm}$ thick, were harvested from the left (LMCA, LAD, LCX) and right coronary (RCA) arteries of each animal. Following standard histology processing, 20-30 serial crosssections, $5 \mu \mathrm{m}$ thick, were cut from paraffin embedded samples by rotary microtome (Microm HM 300, Bio-optica).
Consecutive sections of each arterial segment were stained with both standard (Haematoxylin and Eosin, H\&E) and with Masson thrichrome and Weigert van Gieson (WVG) stains for collagen and elastic laminas respectively, and examined under light microscopy (Olympus BX43) at $4 \mathrm{X}$ to $40 \mathrm{X}$ original magnification. After acquisition by a video system (Olympus D70 camera) interfaced to a computer with dedicated software (Olympus Cell Sens Dimension), image processing and morphometric analysis of each section were performed.

All lesions were classified from type I to VI, according to the current Stary's stages for atherosclerotic plaques of the American Heart Association Committee on Vascular Lesions [18].

Lesion size was expressed in terms of thickness and cross-sectional area. The standard morphometric parameters used to define plaque thickness are the maximal distance from internal elastic lamina to endothelial layer (maximal intimal thickness, IT) and the maximal intima to media thickness ratio (IMT ratio); for plaque area, as coronary cross sections are affected by histologic distortion and shrinkage of the wall [19], we used, in addition to absolute lesion area value $\left(\mathrm{LA}, \mathrm{mm}^{2}\right)$, the ratio of lesion to total vessel wall area (intima +media area surrounded by external elastic lamina)x100 (\% lesion area, \% LA). All four indexes were calculated in each section and used as a quantitative estimate of overall ATS burden by averaging the values of all sections/segments which display pathologic changes in each animal and expressed as Mean IT, Mean IMT ratio, Mean LA and Mean\% LA. The most severe plaque of each case was taken as a marker of maximal coronary ATS progression and its parameters measured and expressed as Max IT, Max IMT ratio, Max LA and Max\% LA. The relative percentage of atheromas (Frequency of Atheromas) (Stary's stage IV or higher) on the total number of lesional segments in each case was also assessed The number of coronary segments analyzed for quantitative assessment of CAD and the incidence of distinct lesion types according to Stary's stages were summarized in Table 1.

\begin{tabular}{|l|l|l|}
\hline & N. & $\%$ \\
\hline All coronary Segments & 135 & 100 \\
\hline Segments with lesions & 113 & 84 \\
\hline Type I & 18 & 16 \\
\hline Type II & 45 & 40 \\
\hline Type III & 14 & 12 \\
\hline Type IV & 11 & 10 \\
\hline Type Va & 14 & 12 \\
\hline Type Vb & 11 & 10 \\
\hline HCD:Hypercholesterolemic Diet & \\
\hline
\end{tabular}

Table 1:Total number of coronary segments analyzed and incidence (number, N. and percentage, \%) of each lesion type in the $6 \mathrm{HCD}$ treated pigs.

\section{Blood sampling}

Blood samples were collected at baseline and at the post-diet time points. Blood cell counts were performed on EDTA-anticoagulated samples by the ADVIA 2120 White Blood Cell Technology System (Siemens Healthcare Diagnostics, Italy) to determine red blood cells 
Citation: Sbrana S, Pelosi G, Puntoni MR, Viglione F, Trivella MG, et al. (2014) Phenotype Changes of Circulating Monocytes in a Hypercholesterolemic Swine Model of Coronary Artery Disease. J Cytol Histol 5: 270. doi:10.4172/2157-7099.1000270

Page 3 of 9

(RBCs), platelets (PLTs) and total white blood cells (WBCs) counts, as well as hemoglobin $(\mathrm{Hb})$ and hematocrit $(\mathrm{Hct})$ levels. Blood smears were May-Grunwald-Giemsa stained and visually evaluated for white blood cell differential counts. The plasma level of high-density lipoprotein (HDL) was quantified by standard enzymatic techniques (Synchron CX9 Pro, Beckman Coulter, Inc., Fullerton, CA, USA). Low-density lipoproteins (LDL) were quantified according to Friedewald formula [20]. Aliquots of plasma obtained from EDTAanticoagulated blood samples centrifuged at $3,000 \mathrm{~g}$ for $10 \mathrm{~min}$ at $4^{\circ} \mathrm{C}$, and immediately frozen at $-80^{\circ} \mathrm{C}$, were used to detect and quantify by ELISA tests the circulating levels of pro- and anti-inflammatory cytokines (IL- 6, TNF- $\alpha$, IFN- $\gamma$, IL-10) and oxidized low-density lipoproteins (oxLDL), as well as of the endothelial adhesion molecule ICAM-1. The minimum detectable concentrations for each cytokine were $3.7 \mathrm{pg} / \mathrm{mL}$ for TNF- $\alpha, 2.03 \mathrm{pg} / \mathrm{mL}$ for IL-6, $6.01 \mathrm{pg} / \mathrm{mL}$ for IFN- $\gamma$ and $3.5 \mathrm{pg} / \mathrm{mL}$ for IL-10.

\section{Flow cytometry analysis}

The evaluated swine monocyte surface markers were: CD172a, CD14, SLA-DR, CD16, CD163, CD18a, CD11a, CD11R3, CD49d, CD29.

Flow cytometry analysis of swine blood monocytes was carried out on EDTA-anticoagulated samples, according to a triple staining procedure already applied by our group to a phenotypical characterization of human circulating monocytes during extracorporeal circulation [21,22]. Briefly, after red blood cells lysis using an ammonium-chloride based solution, the isolated leukocyte suspension were treated as follows: a) primary on ice incubation for 30 min with a purified mouse monoclonal antibody (IgG1 isotype) recognizing the swine pan-myeloid marker CD172a (SWC3); b) washing with cold PBS (BSA 0.5\%) saline solution (staining buffer) by centrifugation at $300 \mathrm{~g}$ for $10 \mathrm{~min}$ at $4^{\circ} \mathrm{C}$ to eliminate the unbound antibody; c) on ice incubation for further $30 \mathrm{~min}$ with a Peridinin Chlorophyll Protein (PerCP)-conjugated rat anti-mouse IgG1 secondary antibody; d) washing and subsequent double staining on ice for $30 \mathrm{~min}$ with a mixtures of Fluorescein Isothiocyanate (FITC)- and Phycoerythrin (PE)-conjugated mouse monoclonal antibodies directed against the porcine monocyte surface molecule combinations CD11a/ CD18a, CD11R3/CD18a, CD49d/CD29, CD14/CD16, CD14/CD163, SLA-DR/CD163; e) final washing and re-suspension in $400 \mu$ of cold staining buffer for flow analysis. Mouse isotype controls were carried out in parallel to quantify the unspecific binding of the main antibodies. Monoclonal antibodies were obtained from Immunotech (Beckman Coulter, Fullerton, CA, USA), Novus Biologicals (Littleton, CO, USA), Serotec (MorphoSys Ltd, Kidlington, UK) and R\&D Systems (Minneapolis, MN, USA). The flow cytometry acquisition process was stopped after 5,000 monocytes, simultaneously gated on the basis of their side scatter (SSC) properties and their bright staining for CD172a (FL3), were collected for each sample (Figure 1).

Flow data analysis was based either on measurement of positive events for each marker (as percentage) or on quantification of its relative amount per cell, in terms of relative fluorescence intensity (RFI), expressed as linear units after subtracting the median value of the isotype-matched control histogram from that corresponding to the specific marker (Figure 1). Moreover, a dual parameter dot-plot of FL2 (CD163) vs. FL1 (CD14) was used to quantify (as percentage) the CD $14^{\text {low }} / \mathrm{CD} 163^{\text {high }}$ blood monocyte fraction (Figure 2), as reported [13]. Flow cytometry analysis was performed with a FACScan instrument and the CellQuest software program (Becton Dickinson, San Jose, CA, USA).

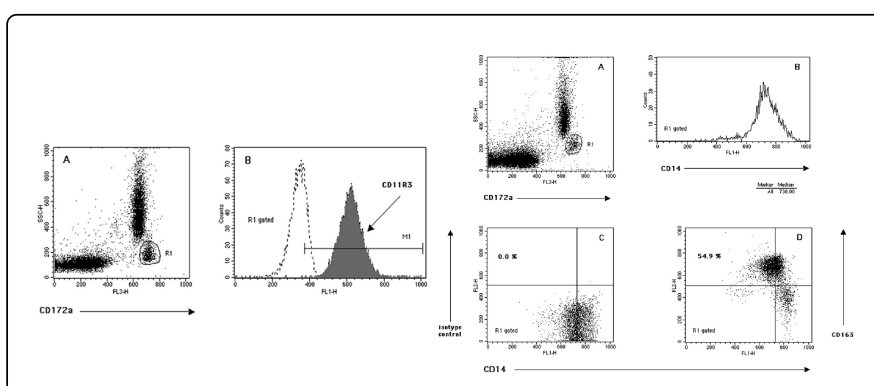

Figure 1: Left panels: Representative example of flow cytometry quantification of the CD11R3 surface marker expression on swine blood monocytes. A: Dual-parameter dot-plot identification of the monocyte cluster (R1) based on side light scatter (SSC) properties and the bright fluorescence for the pan-myeloid marker CD172a (FL3). The R1-derived overlaid histograms subtraction (test histogram-control histogram) (B) has been used to quantify the percentages of positivity for CD11R3 (events in M1, filled histogram) and its relative fluorescence intensity (RFI) (linear scale) with respect to the corresponding isotype controls (dotted histogram). Right panels: Flow cytometry quantification of the circulating CD14 $4^{\text {low }} / \mathrm{CD} 163^{\text {high }}$ monocyte fraction (as percentage). A: The monocytes have been clustered as previously described. Based on the median channel (linear scale) of the R1-derived CD14 fluorescence distribution (FL1, B), a dual-parameter dot-plot quadrant of FL2 vs FL1 has been used to quantify the percentage of CD14 ${ }^{\text {low }} / \mathrm{CD} 163^{\text {high }}$ monocytes (upper left quadrant, D) compared with the corresponding isotype control (upper left quadrant, C).
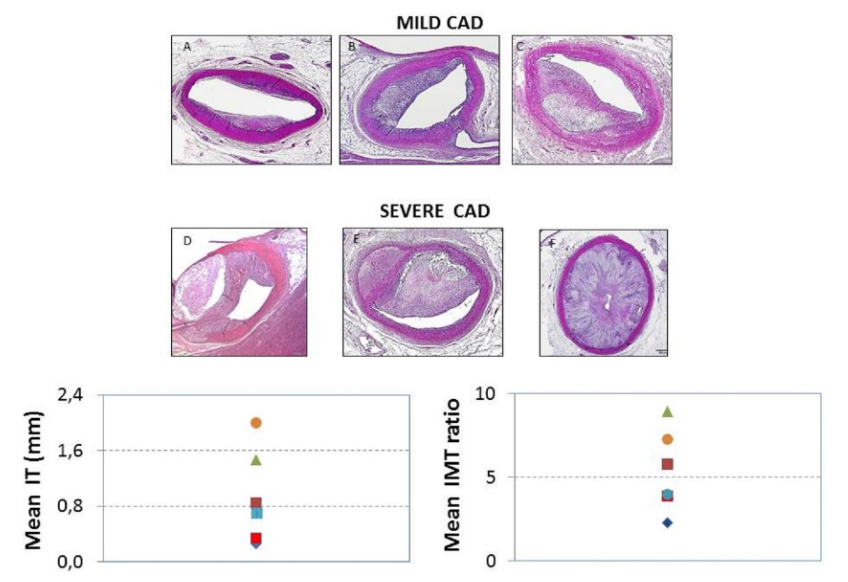

Figure 2:Top panels:Most severe coronary atherosclerotic lesion of each HCD case: pre-atheromas (A, B), type IV initial atheromas (C), fibrofatty (D) and fibrocalcific (E, F) atheromas (H\&E, $2 \mathrm{X}$ original magnification). Bottom panels: Inter-animal range of Mean IT and Mean IMT ratio. Mild and more severe CAD cases can be identified based on these two parameters as well as on the incidence of atheromas, as reported in Table 4. 
Citation: Sbrana S, Pelosi G, Puntoni MR, Viglione F, Trivella MG, et al. (2014) Phenotype Changes of Circulating Monocytes in a Hypercholesterolemic Swine Model of Coronary Artery Disease. J Cytol Histol 5: 270. doi:10.4172/2157-7099.1000270

Page 4 of 9

\section{Statistical analysis}

The mean values of measurements, their standard errors (SEM) and standard deviations (SD), as well as the linear regressions among different groups of data were calculated with the Stat View 5.0 software program (SAS Institute, Cary, NC, USA). The statistical significant differences between groups was determined byFisher's t-test for repeated measurements. A $\mathrm{P}<0.05$ was considered statistically significant.

\section{Results}

\section{Hypercholesterolemic diet (HCD)-induced biochemical changes and hematological modifications}

The animal weight ranged from $30.833 \pm 1.939$ at baseline to 49.167 $\pm 3.081 \mathrm{~kg}$ after 16 weeks $\mathrm{HCD}(\mathrm{P}<0.0001)$. There was a significant post-diet increase of total cholesterol and LDL concentrations compared with baseline values. Post-diet vs. baseline increments of HDL and oxLDLconcentrations, although statistically not significant, were also observed.

An augmentation, even if statistically not significant, of post-diet vs. baseline blood levels of the pro-inflammatory cytokines TNF- $\alpha$ and IL- 6 has been observed. The circulating levels of cytokines IFN- $\gamma$ and IL-10 resulted undetectable, while sICAM-1 level was not significantly changed.

HCD-treated pigs exhibited a slight post-diet decrease of total WBCs count and hematocrit volume compared with baseline values. These findings were associated to a not significant post-diet decrease of RBC counts ( ${ }^{\circ}$ of cells $\left.\times 10^{6} / \mu \mathrm{L}\right)(5.083 \pm 0.192$ vs. $5.755 \pm 0.292$, $\mathrm{P}=0.0507)$ and blood monocyte number ( $\mathrm{n}^{\circ}$ of cells $\left./ \mu \mathrm{L}\right)(850 \pm 99$ vs. $1060 \pm 183, \mathrm{P}=\mathrm{ns}$ ). Table 2 summarizes these results.

\begin{tabular}{|c|c|c|c|}
\hline & Baseline & Post-diet & $\mathbf{P}$ \\
\hline WBC $\left({ }^{*} 1000 \mathrm{n} / \mu \mathrm{L}\right)$ & $12.98 \pm 6.31$ & $10.26 \pm 2.49$ & ns \\
\hline НCT \% & $27.72 \pm 3.91$ & $25.08 \pm 1.16$ & ns \\
\hline Creatinine (mg/dL) & $0.99 \pm 0.32$ & $1.78 \pm 0.57$ & 0.0220 \\
\hline Glucose (mg/dL) & $90.00 \pm 25.59$ & $67.17 \pm 31.73$ & ns \\
\hline Total Cholesterol (mg/dL) & $71.83 \pm 29.03$ & $557.83 \pm 133.73$ & 0.0001 \\
\hline $\mathrm{HDL}(\mathrm{mg} / \mathrm{dL})$ & $24.83 \pm 12.54$ & $33.67 \pm 14.35$ & ns \\
\hline LDL (mg/dL) & $35.10 \pm 17.27$ & $511.17 \pm 133.60$ & 0.0002 \\
\hline Ch tot/HDL ratio & $3.42 \pm 0.65$ & $18.88 \pm 7.08$ & 0.0129 \\
\hline oxLDL (U/L) & $17.75 \pm 6.69$ & $45.50 \pm 43.99$ & ns \\
\hline TG (mg/dL) & $59.00 \pm 50.05$ & $65.17 \pm 53.94$ & ns \\
\hline GOT (IU/L) & $39.50 \pm 29.95$ & $112.00 \pm 113.27$ & ns \\
\hline GPT (IU/L) & $31.33 \pm 6.74$ & $47.83 \pm 8.59$ & 0.0314 \\
\hline Gammaglutamyl-transferase (UI/L) & $33.50 \pm 16.81$ & $74.40 \pm 25.15$ & 0.0368 \\
\hline Apolipoprotein A-I (mg/dL) & $25.96 \pm 12.97$ & $54.65 \pm 6.37$ & 0.0039 \\
\hline sICAM-1 (ng/mL) & $567.88 \pm 330.30$ & $503.61 \pm 175.47$ & ns \\
\hline IL6 (pg/mL) & $89.35 \pm 122.68$ & $128.36 \pm 142.21$ & ns \\
\hline TNFalpha (pg/mL) & $55.40 \pm 28.83$ & $405.30 \pm 847.44$ & ns \\
\hline
\end{tabular}

Table 2:Cumulative quantitative variations $($ Mean $\pm \mathrm{SD}$ ) of biochemical and hematological parameters in the 6 HCD-treated pigs.

\section{Phenotype changes of circulating monocytes after 16 weeks HCD}

The porcine mononuclear phagocyte system, as the human system, is characterized by the expression of several surface molecules [23]. The modulation of these phenotype markers identifies different levels of cellular activation and functional capabilities [24,25].

The cumulative analysis of blood monocyte surface markers revealed a significant post-diet down modulation of CD14 expression, either as percentage of positivity or relative fluorescence intensity (RFI) (Table 2). Moreover, compared with baseline values, a post-diet increase of SLA-DR expression significant in terms of cell positive fraction, was also evident (Table 3).

The post-diet mean value of the $\mathrm{CD} 14^{\text {low }} / \mathrm{CD} 163^{\text {high }}$ monocyte fraction (as percentage) was found to be slightly higher in comparison with baseline data $(45.07 \pm 2.27$ vs. $40.14 \pm 3.16, \mathrm{P}=\mathrm{ns})$. 
Citation: Sbrana S, Pelosi G, Puntoni MR, Viglione F, Trivella MG, et al. (2014) Phenotype Changes of Circulating Monocytes in a Hypercholesterolemic Swine Model of Coronary Artery Disease. J Cytol Histol 5: 270. doi:10.4172/2157-7099.1000270

Page 5 of 9

\begin{tabular}{|c|c|c|c|c|c|}
\hline Marker & $\begin{array}{l}\text { \%Positivity } \\
\text { (Baseline) }\end{array}$ & $\begin{array}{l}\text { \% Positivity } \\
\text { (Post-diet) }\end{array}$ & $\begin{array}{l}\text { RFI } \\
\text { (Baseline) }\end{array}$ & $\begin{array}{l}\text { RFI } \\
\text { (Post-diet) }\end{array}$ & Functional description \\
\hline CD14 & $99.25 \pm 0.228$ & $\begin{array}{l}97.87 \pm 0.516 \\
(P=0.0083)\end{array}$ & $490.75 \pm 10.063$ & $\begin{array}{l}428.91 \pm 16.192 \\
(P=0.0128)\end{array}$ & Monocyte differentiation/maturation antigen \\
\hline CD18a & $99.99 \pm 0.008$ & $\begin{array}{l}99.93 \pm 0.058 \\
(P=n s)\end{array}$ & $628.25 \pm 10.446$ & $\begin{array}{l}638.25 \pm 14.631 \\
(P=n s)\end{array}$ & $\begin{array}{l}\beta \text { chain of the leukocyte integrin complexes } \\
\text { CD11a/CD18 (LFA-1) [ligand for ICAM-1, } \\
\text { ICAM-2, ICAM-3 on endothelial cells] and } \\
\text { CD11b/CD18 (Mac-1) [ligand for ICAM-1 on } \\
\text { endothelial cells] }\end{array}$ \\
\hline CD11a & $99.83 \pm 0.036$ & $\begin{array}{l}99.43 \pm 0.385 \\
(P=n s)\end{array}$ & $412.41 \pm 7.200$ & $\begin{array}{l}390.16 \pm 8.324 \\
(P=n s)\end{array}$ & $\alpha$ chain of the leukocyte integrin complex LFA-1 \\
\hline CD11R3 & $98.98 \pm 0.210$ & $\begin{array}{l}99.01 \pm 0.229 \\
(P=n s)\end{array}$ & $323.33 \pm 13.803$ & $\begin{array}{l}350.00 \pm 17.025 \\
(P=n s)\end{array}$ & $\begin{array}{l}\alpha \text { chain of the leukocyte integrin complex Mac- } 1 \text {, } \\
\text { equivalent to human CD } 11 \mathrm{~b}\end{array}$ \\
\hline CD49d & $99.09 \pm 0.189$ & $\begin{array}{l}99.09 \pm 0.217 \\
(P=n s)\end{array}$ & $323.50 \pm 6.136$ & $\begin{array}{l}305.33 \pm 5.829 \\
(P=n s)\end{array}$ & $\begin{array}{l}\alpha \text { chain of the leukocyte integrin complex } \\
\text { CD49d/CD29 (VLA-4) [ligand for VCAM-1 on } \\
\text { endothelial cells] }\end{array}$ \\
\hline CD29 & $99.96 \pm 0.013$ & $\begin{array}{l}99.93 \pm 0.016 \\
(P=n s)\end{array}$ & $674.33 \pm 12.393$ & $\begin{array}{l}674.75 \pm 24.605 \\
(P=n s)\end{array}$ & $\beta$ chain of the leukocyte integrin complex VLA-4 \\
\hline CD16 & $99.98 \pm 0.010$ & $\begin{array}{l}99.96 \pm 0.018 \\
(P=n s)\end{array}$ & $585.16 \pm 17.155$ & $\begin{array}{l}595.25 \pm 14.180 \\
(P=n s)\end{array}$ & FcyRIII, low affinity receptor for IgG \\
\hline CD163 & $99.94 \pm 0.031$ & $\begin{array}{l}99.20 \pm 0.475 \\
(P=n s)\end{array}$ & $581.27 \pm 42.086$ & $\begin{array}{l}605.45 \pm 33.004 \\
(P=n s)\end{array}$ & Haptoglobin scavenger receptor \\
\hline SLA-DR & $48.26 \pm 2.336$ & $\begin{array}{l}62.06 \pm 5.432 \\
(P=0.0379)\end{array}$ & $249.75 \pm 14.156$ & $\begin{array}{l}284.41 \pm 20.086 \\
(P=n s)\end{array}$ & $\begin{array}{l}\text { Monocyte activation marker, equivalent to } \\
\text { human HLA-DR }\end{array}$ \\
\hline
\end{tabular}

Table 3:Cumulative quantitative variations (Mean \pm SEM) of surface markers expression (as percentage of positivity and RFI) in the $6 \mathrm{HCD}$ treated pigs.Functional description of the markers is reported.

\section{Histology and histomorphometry}

The majority of coronary samples $(84 \%)$ displayedatherosclerotic changes: out of the 113 coronary segments with coronary lesions, type IV (atheroma) to type $\mathrm{V}$ plaques were present in about one third, while type III pre-atheroma or earlier stages of pre-atherosclerotic lesions in the other ones.

\begin{tabular}{|l|l|l|l|}
\hline Histomorphometry & HCD cases(6) & MILD CAD (3) & $\begin{array}{l}\text { SEVERE CAD } \\
\mathbf{( 3 )}\end{array}$ \\
\hline \%Atheromas & $28.67 \pm 17.23$ & $9.00 \pm 6.14$ & $48.33 \pm 20.36$ \\
\hline Mean IT (mm) & $0.59 \pm 0.30$ & $0.36 \pm 0.11$ & $0.84 \pm 0.25$ \\
\hline Mean LA (mm $\left.{ }^{2}\right)$ & $2.40 \pm 1.24$ & $1.03 \pm 0.20$ & $2.85 \pm 1.14$ \\
\hline Mean IMT ratio & $2.64 \pm 1.40$ & $1.19 \pm 0.36$ & $3.49 \pm 1.11$ \\
\hline Mean LA\% & $31.17 \pm 8.25$ & $19.78 \pm 6.11$ & $42.58 \pm 7.60$ \\
\hline SEM:Standard Error of the Mean;CAD:Coronary Artery Disease \\
\hline
\end{tabular}

Table 4: Mean values \pm SEM of most relevant histomorphometric parameters in all cases as well as in the two separate subgroups with different CAD severity.
Top panels of Figure 2 illustrate the spectrum of lesions (maximal lesion of each case) observed in six HCD treated animals. Histomorphometry of coronary lesions confirmed a broad interanimal variability in lesion stage and severity. Mild (3 cases) and more severe ( 3 cases) coronary ATS is observed in HCD treated animals and a lower variability of ATS burden found in each subgroup, as summarized in Table 4 for the relevant morphometric parameters measured.

\section{Associations between monocyte phenotype changes and histomorphometric parameters of ATS burden}

The pre-diet activation state of circulating monocytes, represented by the fraction (percentage) of cells expressing the activation marker SLA-DR (with a CV, coefficient of variation=12\%), significantly correlated with the end-diet values of histopathologic parameters used to quantify coronary ATS spreading, such as Mean IT, and stage of progression (Frequency of Atheromas), as depicted in Figure 3 (left panels). The monocyte percentages of positivity for SLA-DR correlated positively with baseline values of soluble ICAM-1 $\left(\mathrm{P}=0.0191, \mathrm{R}^{2}=0.783\right)$. 

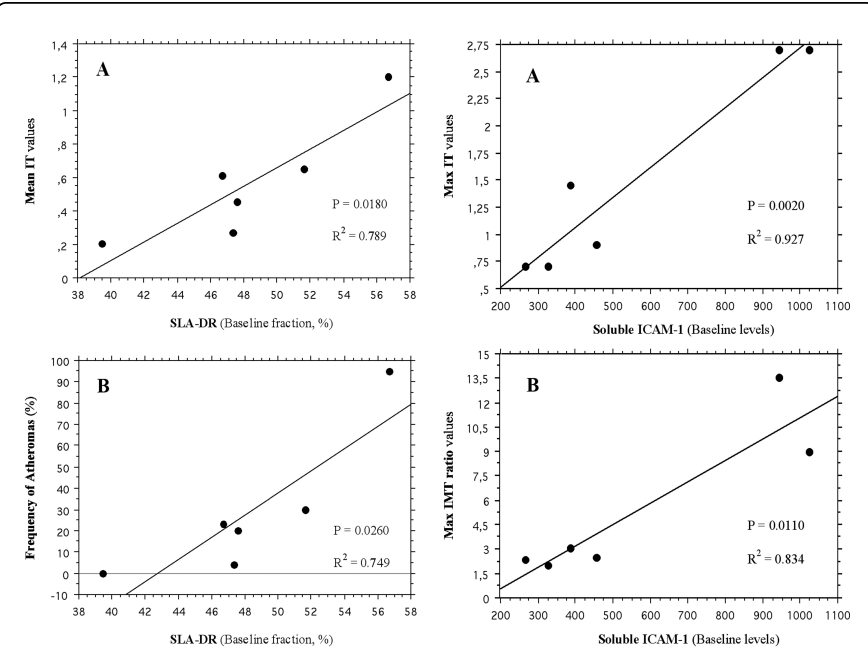

Figure 3:Left panels: Correlation between the baseline fraction (as percentage) of SLA-DR positive monocytes and the corresponding end-diet values of Mean IT (A) and Frequency of Atheromas (B), representative of the coronary ATS extent. Right panels: Correlation between baseline values of soluble ICAM-1 and the corresponding end-diet values of Max IT (A) and Max IMT ratio (B), quantifying the maximal coronary ATS stage.

The pre-diet values of the soluble ICAM-1 showed a high interanimal variability $(\mathrm{CV}=58 \%)$ and significantly correlated both with the end-diet values of histopathologic parameters used to quantify maximal atherosclerotic progression stage, such as max IT and max IMT ratio (Fig. 3, right panels), as well as with average atherosclerotic burden/progression parameters, such as Frequency of Atheromas $\left(\mathrm{P}=0.0395, \mathrm{R}^{2}=0.694\right)$ and Mean IT $\left(\mathrm{P}=0.0292, \mathrm{R}^{2}=0.734\right)$.

A positive correlation was observed between intra-animal variations (end-diet vs. baseline, as percentage) of the relative RFI values of the CD29 integrin subunit and the corresponding histopathologic indexes of maximal ATS progression such as Max IT and Max IMT ratio (Figure 4, panels A and B). A similar behavior has been found for the monocyte $\beta 2$-integrin $\mathrm{CD} 18 \mathrm{a}$, with significant positive regressions between its RFI changes and the histopathologic parameters Max IT and Max IMT ratio (Figure 4, panels C and D).

For each animal, positive significant regressions were observed between ATS stage and the corresponding end-diet RFI values of CD29 $\left(\mathrm{P}=0.0212, \mathrm{R}^{2}=0.772\right)$ and CD18a $\left(\mathrm{P}=0.0231, \mathrm{R}^{2}=0.763\right)$. Similarly, a strongly positive significant relationship was observed between the end-diet CD16 RFI values and the corresponding histopathologic parameters of ATS progression, Max IT $(\mathrm{P}=0.0093$, $\left.\mathrm{R}^{2}=0.847\right)$ and Max IMT ratio $\left(\mathrm{P}=0.0020, \mathrm{R}^{2}=0.927\right)$.

Moreover, we observed that the baseline values of the CD14 $4^{\text {low }}$ CD163 high monocyte fraction $(\mathrm{CV}=19 \%)$ showed positive but not significant trends with the histopathologic parameters Mean IT $\left(\mathrm{P}=0.0530, \mathrm{R}^{2}=0.649\right)$ and Frequency of Atheromas $(\mathrm{P}=0.0823$, $\mathrm{R}^{2=} 0.571$ ). On the other hand, the individual variations (end-diet vs. baseline, as percentage) of this monocyte subset showed clearly inverse trends, even if statistically not significant, with all histopathologic parameters (data not shown). Finally, a significant positive linear regression $\left(\mathrm{P}=0.0100, \mathrm{R}^{2}=0.842\right)$ was observed between individual
HDL variations and the corresponding changes (as percentage) of the $\mathrm{CD} 14^{\text {low }} / \mathrm{CD} 163^{\text {high }}$ monocyte fraction.
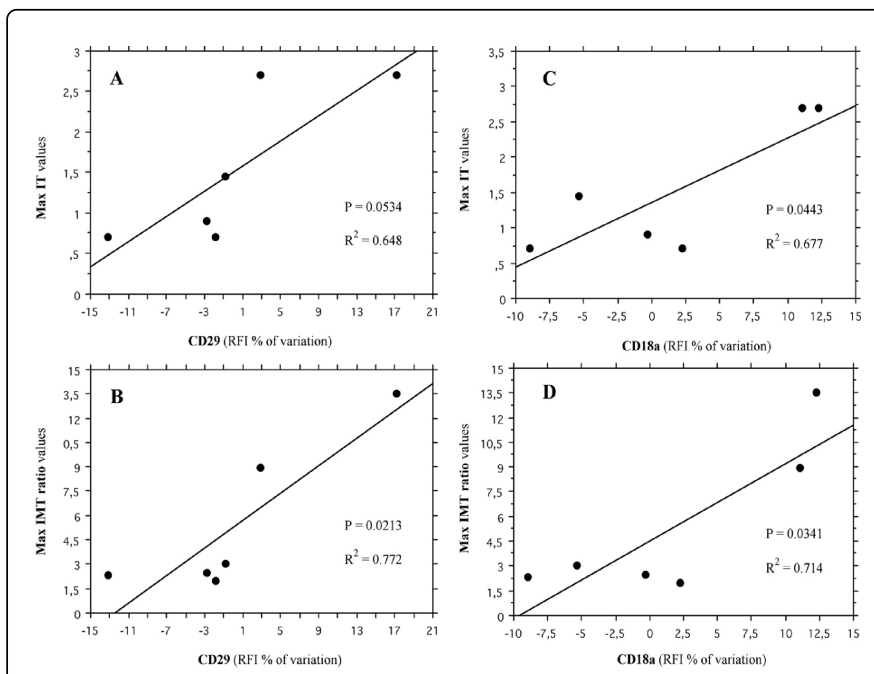

Figure 4: Relationships between intra-animal variations (end-diet vs. baseline, as percentage) of the relative fluorescence intensity (RFI) of the monocyte integrin molecules CD29 and CD18a and the corresponding end-diet values of Max IT (panels A and C) and Max IMT ratio (panels B and D).

\section{Discussion}

\section{Cumulative diet-induced blood monocytes phenotype changes and characterization of coronary ATS burden/ progression}

In the present study we employed a swine model of CAD in order to evaluate, by flow cytometry, HCD-induced phenotype modifications of circulating monocytes and their relationship with severity and extent of atherosclerotic lesions assessed by histomorphomety.

To our knowledge, this study design represents an original link between coronary histomorphometry and blood immune cell flow cytometry data in a swine model of experimentally induced CAD. In particular, once associated with post-diet histopathologic parameters quantifying either ATS extent in the entire coronary tree (Mean IT and Mean IMT ratio), or the features of most advanced localized atherosclerotic lesions (Max IT and Max IMT ratio), these data could help in clarifying the relationship between innate immune-mediated mechanisms and ATS initiation and progression [26].

Following 4 months HCD, we observed a decreased number of circulating monocytes associated with a down-modulated expression of monocyte differentiation antigen CD14 and an increased fraction of cells expressing the activation marker SLA-DR. According to previous studies demonstrating that porcine blood monocytes differentiate towards a more mature macrophage-like phenotype characterized by up-regulation of SLA-DR and down-regulation of CD14 [25], our findings can reflect a diet-induced macrophage-like maturation of circulating monocytes and their increased retention into coronary arterial sites prone to develop atherosclerotic lesions. 


\section{Blood monocyte phenotype and endothelial markers as predictors of ATS burden/progression}

The results of our study suggest the capability of some baseline (pre-diet) cellular and soluble markers to predict CAD severity and extent of lesions in the swine model of HCD-induced coronary ATS. In fact, for each animal, the pre-diet values of SLA-DR positive monocyte fraction resulted significantly correlated to end-diet coronary ATS stage and extent, independently of blood level variations of the known pro-atherogenic (oxLDL) and athero-protective (HDL) lipoproteins $[27,28]$.

Similarly, the pre-diet levels of sICAM-1, an endothelial activation marker [29,30], significantly correlated with final average ATS burden and stage of disease, in agreement with previous clinical studies relating basal levels of sICAM-1 and ATS progression [31,32].

Moreover, pre-diet sICAM-1 levels are tightly related to monocyte positivity of the activation marker SLA-DR, which is found to correlate, in its turn, with baseline monocyte expression of CD29 integrin subunit. All above observations might support the hypothesis of a monocyte-induced endothelial dysfunction. The CD29 subunit forms, in association with the CD49d a4-integrin subunit, the very late antigen-4 (VLA-4), which represents the main integrin complex involved in monocyte adhesion to endothelial cell [33,34]. The importance of the CD29 molecule expression level (in terms of RFI) on blood monocytes in influencing ATS progression has been further confirmed for the first time in this study by the positive correlations between individual RFI variations of this integrin subunit expression and coronary ATS severity and extent. A similar behavior is found for the monocyte $\beta 2$-integrin $\mathrm{CD} 18 \mathrm{a}$ that shows significant positive relationships with ATS stage.

All these findings suggest that pre-diet functional state of circulating monocytes could influence baseline endothelial activation state itself, probably as a consequence of an individually modulated surface expression of adhesion molecules and/or co-stimulatory receptors able to bind to specific endothelial ligands [35]. No previous experimental or clinical studies have proposed such a mechanism of ATS initiation.

\section{Pathophysiologic and clinical hypothesis}

Previous human studies have demonstrated the usefulness of flow cytometry quantification of integrin molecules expression on circulating monocytes, from a single blood drawing, in predicting the progression of coronary and peripheral atherosclerotic arterial disease under different clinical conditions [30,36]. Under this aspect, the significantly positive relationships observed for the first time in our study between ATS stage and corresponding end-diet RFI values of CD29 and CD18a propose these integrin molecules as candidate markers to monitor ATS coronary lesion progression.

Baseline fractions of SLA-DR positive monocytes have been found to marginally correlate also with corresponding values of CD16 expression. The CD16 (or Fc $\gamma$ RIII) is the low-affinity receptor for the IgG Fc portion and its higher expression on swine monocyte lineages indicates an activation state and a more mature stage of differentiation towards macrophages [37]. Previous clinical studies demonstrated the importance of CD16 quantification on distinct monocyte subsets in predicting adverse cardiovascular events in subjects recruited for elective coronary angiography [38]. However, to our knowledge, neither clinical nor experimental studies evaluated, so far, the relationships between CD16 expression level (quantified as RFI) on all circulating monocytes and coronary ATS evolution. In our study, for the first time, the end-diet CD16 RFI values derived from all blood monocytes have been shown to correlate strongly with ATS maximal severity, suggesting a relevant association between this monocyte activation marker and ATS progression. On this basis, the above mentioned pro-adhesive and activation monocyte surface antigens could represent not only useful markers to monitor ATS progression, but also potential targets for newly developed immune-based therapies.

Pig monocytes have been divided into two major subpopulations on the basis of the CD163 marker expression [24]. As in humans, the CD163 expression in pigs is restricted to monocyte/macrophages and clearly defines a distinctive monocyte subset $[13,39]$. In particular, the simultaneous flow cytometry analysis of CD14 and CD163 expression on pig blood monocytes showed that CD163 is expressed on the CD14 ${ }^{\text {low }}$ cell subset at higher level than on CD14high monocytes [13]. We quantified the CD172-derived $\mathrm{CD} 14^{\text {low }} / \mathrm{CD} 163^{\text {high }}$ monocyte fraction (as percentage) by using a CD14 fluorescence histogrambased median channel and a bivariate fluorescence dot-plot of CD163 vs. CD14, as already reported [13]. We found that, for each animal, the baseline fraction of $\mathrm{CD} 14^{\text {low }} / \mathrm{CD} 163^{\text {high }}$ monocytes correlated significantly with the corresponding percentages of positivity of other activation markers, in particular CD16 and SLA-DR, on all blood monocytes. Moreover, the presence of a positive significant relationship between the intra-animal HDL variations and the corresponding changes of the CD14 $4^{\text {low }} / \mathrm{CD} 163^{\text {high }}$ monocyte fraction, which are inversely related to the histological features of coronary ATS burden, suggests the hypothesis of a HDL-induced maturation of swine blood monocytes towards a CD $14^{\text {low }} / \mathrm{CD} 163^{\text {high }}$ phenotype that could act in an athero-protective manner, probably as a consequence of a prevalent M2 functional polarization of this subset [40,41].

\section{Limits of the study}

The main study limitation is the low number of studied animals, which restricts the statistical robustness of the relationship between circulating monocyte markers expression and HCD-induced histomorphometric indexes of CAD.

Another limitation is represented by the lack of quantification of macrophages and foam-cells infiltration into ATS lesions and the correlation with the expression level of circulating monocyte markers. However, preliminary histological observations evidenced a low macrophage/foam cell infiltration into coronary lesions after 4 months of $\mathrm{HCD}$, probably reflecting a reduction of monocyte trafficking into ATS coronary lesions. Another study limitation is the absence of blood monocyte chemokine receptors expression evaluation (in particular of CCR2, CCR5 and CX3CR1). This limitation, actually due to the lack of a panel of commercially available monoclonal antibodies specific for the swine chemokine receptors, will represent a future challenge to better understand the role of monocyte trafficking inplaque formation and progression in this experimental model. Under a translational viewpoint, the recent evidence of a significant correlation between carotid plaque inflammation, as assessed by [18F]-fluorodeoxyglucose positron emission/computer tomography technique,and the intraplaque CD68 positive cell content [42], raises the appealing possibility of a non-invasive evaluation of monocyte contribution to ATS plaque progression/evolution in humans. 


\section{Conclusions}

Taken together, our preliminary data suggest the potential relevance of circulating monocyte phenotype features in predicting CAD stage and progression, assessed by histology, in a swine model of experimentally induced ATS.In agreement with human clinical trials, demonstrating the importance of the circulating immune effector characterization in predicting cardiovascular disease outcome [43], our experimental findings, albeit preliminary, support a role of the circulating monocyte functional state in coronary plaque initiation and early progression, providing the basis for a better knowledge of immune cell contribution to CAD development in humans.

\section{Acknowledgements}

This study was supported by grant from EU FP7-ICT-2007 ARTreat project "Multi-level patient-specific artery and atherogenesis model for outcome prediction, decision support treatment, and virtual hand-on training" (grant agreement 224297).

We are grateful to Dr. Silvia Rocchiccioli, $\mathrm{PhD}$, for her comments and suggestions in the Discussion of results.

\section{References}

1. Hansson GK, Robertson AK, Söderberg-Nauclér C (2006) Inflammation and atherosclerosis.Annu Rev Pathol 1: 297-329.

2. Skålén $\mathrm{K}$, Gustafsson $\mathrm{M}$, Rydberg EK, Hultén LM, Wiklund $\mathrm{O}$, et al. (2002) Subendothelial retention of atherogenic lipoproteins in early atherosclerosis.Nature 417: 750-754.

3. Muller WA (2003) Leukocyte-endothelial-cell interactions in leukocyte transmigration and the inflammatory response.Trends Immunol 24 327-334.

4. Charo IF, Taubman MB (2004) Chemokines in the pathogenesis of vascular disease.Circ Res 95: 858-866.

5. Dai G, Kaazempur-Mofrad MR, Natarajan S, Zhang Y, Vaughn S, et al (2004) Distinct endothelial phenotype evoked by arterial waveforms derived from atherosclerosis-susceptible and -resistant regions of human vasculature. Proc Natl Acad Sci USA 101: 14871-14876.

6. Mestas J, Ley K (2008) Monocyte-endothelial cell interactions in the development of atherosclerosis.Trends Cardiovasc Med 18: 228-232.

7. Moore KJ, Tabas I (2011) Macrophages in the pathogenesis of atherosclerosis.Cell 145: 341-355.

8. Ingersoll MA, Platt AM, Potteaux S, Randolph GJ (2011) Monocyte trafficking in acute and chronic inflammation.Trends Immunol 32: 470-477.

9. Gordon S, Taylor PR (2005) Monocyte and macrophage heterogeneity.Nat Rev Immunol 5: 953-964.

10. Ziegler-Heitbrock L, Ancuta P, Crowe S, Dalod M, Grau V, et al. (2010) Nomenclature of monocytes and dendritic cells in blood.Blood 116: e74-80.

11. Geissmann F, Jung S, Littman DR (2003) Blood monocytes consist of two principal subsets with distinct migratory properties.Immunity 19: 71-82.

12. Swirski FK, Libby P, Aikawa E, Alcaide P, Luscinskas FW, et al. (2007) Ly-6Chi monocytes dominate hypercholesterolemia-associated monocytosis and give rise to macrophages in atheromata.J Clin Invest 117: 195-205.

13. Fairbairn L, Kapetanovic R, Sester DP, Hume DA (2011) The mononuclear phagocyte system of the pig as a model for understanding human innate immunity and disease.J Leukoc Biol 89: 855-871.

14. Busnelli M, Froio A, Bacci ML, Giunti M, Cerrito MG, et al. (2009) Pathogenetic role of hypercholesterolemia in a novel preclinical model of vascular injury in pigs.Atherosclerosis 207: 384-390.
15. Perrault LP, Mahlberg F, Breugnot C, Bidouard JP, Villeneuve N, et al. (2000) Hypercholesterolemia increases coronary endothelial dysfunction, lipid content, and accelerated atherosclerosis after heart transplantation. Arterioscler Thromb Vasc Biol 20: 721-727.

16. Recchia D, Abendschein DR, Saffitz JE, Wickline SA (1995) The biologic behavior of balloon hyperinflation-induced arterial lesions in hypercholesterolemic pigs depends on the presence of foam cells.Arterioscler Thromb Vasc Biol 15: 924-929.

17. Geeraert B, De Keyzer D, Davey PC, Crombé F, Benhabilès N, et al (2007) Oxidized low-density lipoprotein-induced expression of ABCA1 in blood monocytes precedes coronary atherosclerosis and is associated with plaque complexity and hypercholesterolemic pigs. J Thromb Haemost 5: 2529-2536

18. Stary HC, Blankenhorn DH, Chandler AB, Glagov S, Insull W Jr, et al. (1992) A definition of the intima of human arteries and of its atherosclerosis-prone regions. A report from the Committee on Vascular Lesions of the Council on Arteriosclerosis, American Heart Associaton. Circulation 85: 391-405.

19. Loraine Lowder M, Li S, Carnell PH, Vito RP (2007) Correction of distortion of histologic sections of arteries.J Biomech 40: 445-450.

20. Friedewald WT, Levy RI, Fredrickson DS (1972) Estimation of the concentration of low-density lipoprotein cholesterol in plasma, without use of the preparative ultracentrifuge.Clin Chem 18: 499-502.

21. Sbrana S, Parri MS, De Filippis R, Gianetti J, Clerico A (2004) Monitoring of monocyte functional state after extracorporeal circulation: a flow cytometry study.Cytometry B Clin Cytom 58: 17-24.

22. Sbrana S, Bevilacqua S, Buffa M, Spiller D, Parri MS, et al. (2005) Postreperfusion changes of monocyte function in coronary blood after extracorporeal circulation. Cytometry B Clin Cytom 65: 14-21.

23. Chamorro S, Revilla C, Alvarez B, López-Fuertes L, Ezquerra A, et al. (2000) Phenotypic characterization of monocyte subpopulations in the pig.Immunobiology 202: 82-93.

24. Sánchez C, Doménech N, Vázquez J, Alonso F, Ezquerra A, et al. (1999) The porcine 2A10 antigen is homologous to human CD163 and related to macrophage differentiation.J Immunol 162: 5230-5237.

25. Chamorro S, Revilla C, Alvarez B, Alonso F, Ezquerra A, et al. (2005) Phenotypic and functional heterogeneity of porcine blood monocytes and its relation with maturation.Immunology 114: 63-71.

26. Saha P, Modarai B, Humphries J, Mattock K, Waltham M, et al. (2009) The monocyte/macrophage as a therapeutic target in atherosclerosis.Curr Opin Pharmacol 9: 109-118.

27. Tellez A, Schuster DS, Alviar C, López-Berenstein G, Sanguino A, et al. (2011) Intramural coronary lipid injection induces atheromatous lesions expressing proinflammatory chemokines: implications for the development of a porcine model of atherosclerosis. Cardiovac Revasc Med 12: 304-311.

28. Sala F1, Catapano AL, Norata GD (2012) High density lipoproteins and atherosclerosis: emerging aspects.J Geriatr Cardiol 9: 401-407.

29. Gearing AJ, Newman W (1993) Circulating adhesion molecules in disease.Immunol Today 14: 506-512.

30. Peschel T, Niebauer J (2003) Role of pro-atherogenic adhesion molecules and inflammatory cytokines in patients with coronary artery disease and diabetes mellitus type 2.Cytometry B Clin Cytom 53: 78-85.

31. Södergren A, Karp K, Boman K, Eriksson C, Lundström E, et al. (2010) Atherosclerosis in early rheumatoid arthritis: very early endothelial activation and rapid progression of intima media thickness.Arthritis Res Ther 12: R158.

32. Chia MC (1998) The role of adhesion molecules in atherosclerosis.Crit Rev Clin Lab Sci 35: 573-602.

33. Cybulsky MI, Gimbrone MA Jr (1991) Endothelial expression of a mononuclear leukocyte adhesion molecule during atherogenesis.Science 251: 788-791.

34. Alon R, Kassner PD, Carr MW, Finger EB, Hemler ME, et al. (1995) The integrin VLA-4 supports tethering and rolling in flow on VCAM-1.J Cell Biol 128: 1243-1253. 
Citation: Sbrana S, Pelosi G, Puntoni MR, Viglione F, Trivella MG, et al. (2014) Phenotype Changes of Circulating Monocytes in a Hypercholesterolemic Swine Model of Coronary Artery Disease. J Cytol Histol 5: 270. doi:10.4172/2157-7099.1000270

Page 9 of 9

35. Hassan GS, Merhi Y, Mourad WM (2009) CD154 and its receptors in inflammatory vascular pathologies.Trends Immunol 30: 165-172.

36. Rahimi K, Maerz HK, Zotz RJ, Tárnok A (2003) Pre-procedural expression of Mac-1 and LFA-1 on leukocytes for prediction of late restenosis and their possible correlation with advanced coronary artery disease.Cytometry B Clin Cytom 53: 63-69.

37. Ezquerra A, Revilla C, Alvarez B, Pérez C, Alonso F, et al. (2009) Porcine myelomonocytic markers and cell populations.Dev Comp Immunol 33: 284-298.

38. Rogacev KS, Cremers B, Zawada AM, Seiler S, Binder N, et al. (2012) CD14++CD16+ monocytes independently predict cardiovascular events. A cohort study of 951 patients referred for elective coronary angiography. J Am Coll Cardiol 60: 1512-1520.

39. Chamorro S, Revilla C, Gómez N, Alvarez B, Alonso F, et al. (2004) In vitro differentiation of porcine blood CD163- and CD163+ monocytes into functional dendritic cells.Immunobiology 209: 57-65.
40. Martinez FO, Helming L, Gordon S (2009) Alternative activation of macrophages: an immunologic functional perspective.Annu Rev Immunol 27: 451-483.

41. Moore KJ, Sheedy FJ, Fisher EA (2013) Macrophages in atherosclerosis: a dynamic balance.Nat Rev Immunol 13: 709-721.

42. Niccoli Asabella A, Ciccone MM, Cortese F, Scicchitano P, Gesualdo M, et al. (2014) Higher reliability of 18F-FDG target background ratio compared to standardized uptake value in vulnerable carotid plaque detection: a pilot study.Ann Nucl Med 28: 571-579.

43. Hoefer IE, Sels JW, Jukema JW, Bergheanu S, Biessen E, et al. (2013) Circulating cells as predictors of secondary manifestations of cardiovascular disease: design of the CIRCULATING CELLS study. Clin Res Cardiol 102: 847-856. 